\title{
Qualidade de Vida Relacionada à Carga de Trabalho dos Profissionais de Saúde com enfoque nos problemas desencadeados
}

\author{
Renata Silva Gomes $^{1}$; Jaqueline Ferraz Rodrigues Coqueiro ${ }^{2}$
}

\begin{abstract}
Resumo: A qualidade de vida vem sendo avaliada nas diversas modalidades de pesquisa e em se tratando desta, voltada para os profissionais de saúde, torna-se ainda mais relevante os estudos, devido à complexidade existente na vida destes trabalhadores, pois lidam com diversas situações que acabam deixando-os expostos aos fatores de risco que envolve a profissão. Buscando desvendar o cotidiano dos trabalhadores dessa área, este estudo apresentou como objetivo geral identificar de que forma a falta da qualidade de vida pode afetar a vida dos profissionais de saúde e como objetivos específicos verificar se existe alguma medida preventiva que pode ser aplicada para os mesmos. Este estudo classifica-se como qualitativo, quanto aos objetivos, descritivo e exploratório com procedimento técnico de pesquisa de campo. Foi realizada uma pesquisa com 50 profissionais de saúde, sem distinção de profissão. Com a coleta das informações, foi possível identificar que os profissionais de saúde são pouco valorizados. A sua carga de trabalho é excessiva e o fato de não terem hora estipulada para trabalhar, demonstra ainda mais o quão estes devem ser valorizados pelas instituições de saúde. Contudo, não foi isso que ficou provado neste estudo. Com base nas informações analisadas é possível afirmar que o objetivo proposto para esse estudo foi alcançado.
\end{abstract}

Palavras chave: Qualidade de vida. Profissionais de Saúde. Carga Horária de Trabalho.

\section{Quality of Life Related to the Workload of Health Professionals focusing on the problems triggered}

\begin{abstract}
The quality of life has been evaluated in the different research modalities and in the case of this one, focused on the health professionals, it becomes even more relevant the studies, due to the complexity existing in the life of these workers, since they deal with several situations that end up Leaving them exposed to the risk factors that involve the profession. This study aimed to find out how the lack of quality of life can affect the life of health professionals and as specific objectives to verify if there is any preventive measure that can be applied to them. This study is classified as qualitative, regarding the objectives, descriptive and exploratory with technical field research. A survey was conducted with 50 health professionals, without distinction of profession. With the collection of information, it was possible to identify that health professionals are undervalued. Their workload is excessive, and the fact that they do not have a set time to work, further demonstrates how these should be valued by health institutions. However, this was not proven in this study. Based on the information analyzed, it is possible to affirm that the objectives proposed for this study were reached.
\end{abstract}

Keywords: Quality of life. Health professionals. Workload.

\footnotetext{
${ }^{1}$ Faculdade Independente do Nordeste - FAINOR. E-mail: rennat_101@hotmail.com.

${ }^{2}$ Graduação em Farmácia pela Universidade Tiradentes, Mestre em Planejamento Ambiental pela Universidade Católica do Salvador, UCSAL. Especialista em Saúde Pública com complementação em Magistério Superior, especialista em Saúde Mental pela Universidade Federal do Rio de Janeiro e em MBA em Auditoria em Serviços de Saúde. Atualmente é Farmacêutica da Vigilância Sanitária da Prefeitura Municipal de Vitória da Conquista, BA. Coordenadora do Curso de Farmácia da Faculdade Independente do Nordeste, FAINOR.
} 
Id on Line Revista Multidisciplinar e de Psicologia

Id on Line Multidisciplinary and Psycology Journal

\section{Introdução}

A expressão qualidade de vida pode vir acompanhada de várias definições e significados, e alguns destes podem variar conforme a interpretação individualizada do termo, devido ao grande número de fatores que podem interferir na definição mais exata, pois, tratase também de um termo com padrões bem subjetivos, ou seja, o que é qualidade de vida para um, pode não ser pra outro. Além disso, existem algumas definições que estão relacionadas à boa condição de saúde, bem estar físico, funcional, emocional e mental. No entanto Bastos (2011) afirma que de acordo a Organização Mundial da Saúde - OMS o conceito de qualidade de vida pode apresentar-se de formas, dependendo também do modo perceptivo existente em cada um.

Para a OMS a saúde pode ser definida como sendo "um estado de completo bem-estar físico, mental e social e não somente ausência de afeções e enfermidades”. Depois das doenças que surgiram com o passar dos anos, a saúde tem sido mais valorizada por grande parte da população. A saúde é um direito fundamental que deve ser oferecida a todas as pessoas sem distinção de raça, de religião ou condição socioeconômica, sendo, portanto considerada um bem de valor. Na década de 60 o ser humano era considerado uma pessoa saudável quando tinha condições físicas e mentais totalmente equilibradas. Hoje, ela é conceituada de maneira muito mais abrangente, ou seja, o fato de como a pessoa vive assim como suas condições de trabalho, também podem ser fatores determinantes para dizer se ele possui saúde ou não, principalmente nas relações existentes no trabalho e com a comunidade (OMS, 2016 p. 01).

Para Pereira (2012) a qualidade de vida pode ser definida como sendo contextos individualizados que giram em torno dos valores culturais e morais existentes em cada um, buscando sempre alcançar os objetivos e as expectativas criados, a fim de enfrentar as preocupações existentes em todo o cotidiano. Contudo, o autor ainda afirma que ao se falar em qualidade de vida, algumas características devem ser observadas, tais como a escolaridade, condições de moradia e desenvolvimento de algumas doenças, onde estas podem interferir diretamente no conceito e na subjetividade que gira em torno à vida de cada um.

É crescente a preocupação com questões relacionadas à qualidade de vida assim como as diversas transformações ocorridas em todo o mundo, onde estas repercutem diretamente na saúde dos trabalhadores de uma forma geral, tanto pelas incorporações de novas tecnologias, 
Id on Line Revista Multidisciplinar e de Psicologia

Id on Line Multidisciplinary and Psycology Journal

quanto pela intensificação laboral. Com a diversificação de atividades o acompanhando todas as transformações existentes no mundo, fez com que o individuo deixasse de focar também na qualidade de vida, mesmo que esse termo seja algo muito subjetivo, incluindo condições variáveis de saúde física, estado psicológico, nível de dependência, família, formação, dinheiro, crenças religiosas, otimismo e toda uma estrutura social (ELVIRA, 2013).

Essas transformações decorrentes das atividades diárias podem desencadear grandes problemas e transtornos relacionados com o homem e o seu trabalho provocando o surgimento de alterações na saúde do profissional, devido à carga horaria de trabalho que por sua vez, necessita passar por uma reorganização, a fim de atender todas as necessidades de cada profissão (ELIAS, 2006).

A qualidade de vida de um profissional pode ser influenciada pelas características individuais como, renda, moradia, relações sociais e carga horaria de trabalho. Toda essa particularidade requer uma atenção pessoal, uma vez que a responsabilidade de se organizar em relação à toda e qualquer atividade a ser exercida é individual. Visto o exposto surgiu à pergunta norteadora desta pesquisa: quais os problemas de saúde desencadeados para os profissionais de saúde devido à carga horária de trabalho, no município de Vitória da Conquista- BA?

Esta pesquisa apresenta como objetivo geral identificar quais os problemas e saúde desencadeados para os profissionais de saúde devido à carga horária excessiva e de que forma a tais problemas podem influenciar na qualidade de vida dos mesmos e como objetivo específico verificar se existe alguma medida preventiva que pode ser aplicada para com esses profissionais.

Esse trabalho é relevante para toda a sociedade, pois irá demonstrar dados sobre a realidade enfrentada pela classe trabalhadora da saúde no mercado de trabalho, assim como para os profissionais que estão adentrando na atividade, servindo como instrumento de estudo. Justifica-se também pela necessidade de entender quais as sequelas e patologias desencadeadas e deixadas pela extensa jornada de trabalho exercida pelos profissionais da área de saúde, foco desta pesquisa, procurando assim entender de que forma alguns destes possam ser sanados. É importante também, pois irá fornecer conhecimento sobre as atuais exigências do mercado de trabalho, possibilitando assim uma adequação mais precisa da assistência para com esses profissionais. 
Id on Line Revista Multidisciplinar e de Psicologia

Id on Line Multidisciplinary and Psycology Journal

\section{Metodologia}

Este estudo se enquadra como pesquisa de cunho qualitativo. Quanto a seus objetivos, classifica-se como descritiva e exploratória e do ponto de vista do procedimento técnico a pesquisa é caracterizada como pesquisa de campo visto que este tipo de estudo tem o intuito de proporcionar maior validade dos resultados principalmente quando se trata de um estudo de cunho, qualitativo (POLIT; BECK; HUNGLER, 2004).

Este estudo foi realizado em Vitória da Conquista - Bahia e os participantes foram 50 profissionais de saúde. A amostra utilizada foi a não probabilística por conveniência, pois, seleciona elementos mais acessíveis de uma determinada população, sendo essa também uma técnica de amostragem importante na definição de uma pesquisa científica (GIL, 2010). Gil (2010) complementa afirmando que a escolha da técnica de amostragem é uma etapa importante do método científico de pesquisa.

Como técnica de escolha optou-se por uma entrevista no qual abordaram questões que giravam em torno dos objetivos propostos pela pesquisa. A entrevista é uma técnica de pesquisa que visa obter informações de interesse a uma investigação, onde o pesquisador formula perguntas orientadas, com um objetivo definido, frente a frente com o respondente e dentro de uma interação social (GIL, 2008).

Um fato que deve ser abordado é que foi feito um recorte dos materiais aprovados pelo Comitê de Ética em pesquisa que se encontram em anexo dentro deste estudo.

Todos os participantes da pesquisa foram devidamente esclarecidos e orientados quanto aos objetivos do trabalho, ficando livres para participar. Os que aceitaram participar assinaram o Termo de Consentimento Livre e Esclarecido - TCLE (APÊNDICE B) sendo respeitados os princípios éticos que constam na resolução 466/12 do Conselho Nacional de Saúde - CNS, que define as diretrizes e normas regulamentadoras de pesquisas envolvendo seres humanos. A pesquisa será enviada após as suas considerações finais para o Comitê de Ética e Pesquisa CEP desta mesma Instituição de ensino. 
Id on Line Revista Multidisciplinar e de Psicologia

Id on Line Multidisciplinary and Psycology Journal

\section{Análise e Discussões}

Neste capítulo serão abordados e analisados todos os resultados obtidos através da pesquisa de campo tendo como a finalidade de obter uma melhor compreensão das respostas dos participantes da pesquisa.

A qualidade de vida do trabalhador da saúde acaba sendo alterada por todas as necessidades exigidas pela profissão, desencadeando distúrbios digestivos e sintomas de fadiga. A promoção da qualidade de vida dos trabalhadores que atuam em grandes períodos envolve um ambiente adequado, estabilidade, boa renumeração, oportunidade de crescimento, entre outras. Atitudes que identifiquem a satisfação do profissional são importantes não apenas para o trabalhador, mas por fornecer atendimento de qualidade à sociedade em geral (NEVES, et. al. 2010).

Devido as grandes dificuldades encontradas pelo homem, devido todos os problemas que andam acometendo no mundo, o trabalhador encontra-se em muitas das vezes obrigados a modificar o valor que é dado a algumas necessidades, ou seja, prioriza em sua grande maioria algo que lhe forneça um retorno financeiro deixando de lado, muitas vezes a sua qualidade de vida. Se tratando da saúde e da qualidade de vida parecia obvio a escolha da saúde como um fator essencial a ser considerado. Porém essa afirmativa não condiz com a realidade, pois muitos nem se quer conhecem ou entendem o verdadeiro sentido e conceito de se ter qualidade de vida, destacando somente o dinheiro como fator primordial para sua vida (JUNIOR, et. al. 2006).

Com alguns estudos que vem sendo realizados, foi possível identificar que existe uma proporção muito grande de profissionais de saúde apresentando estresse, problemas emocionais, insatisfação no trabalho, fatores esses que interferem diretamente na saúde do trabalhador. Além desses problemas existem as questões que envolvem o ambiente de trabalho no qual estão inseridos, a indisponibilidade de profissionais na proporção adequada, necessitando em alguns casos, a técnica de negociação dos mesmos para com as instituições nas quais está inserida, solução esta que é administrada na tentativa de ser sanada (COREN, 2014). 
Id on Line Revista Multidisciplinar e de Psicologia

Id on Line Multidisciplinary and Psycology Journal

TABELA 01: Perfil dos profissionais de saúde entrevistados

\begin{tabular}{|c|c|c|}
\hline VARIÁVEL & $\mathbf{N}$ & $\%$ \\
\hline Sexo & & \\
\hline Feminino & 44 & $88 \%$ \\
\hline Masculino & 06 & $12 \%$ \\
\hline Estado civil & & \\
\hline Casado & 31 & $62 \%$ \\
\hline Solteiro & 01 & $2 \%$ \\
\hline Divorciado & 18 & $36 \%$ \\
\hline Escolaridade & & \\
\hline Ensino Fundamental Incompleto & 01 & $4 \%$ \\
\hline Ensino Fundamental Completo & 03 & $6 \%$ \\
\hline Ensino Médio Incompleto & 03 & $6 \%$ \\
\hline Ensino Médio Completo & 20 & $40 \%$ \\
\hline Ensino Superior Incompleto & 05 & $10 \%$ \\
\hline Ensino Superior Completo & 17 & $34 \%$ \\
\hline Idade & & \\
\hline 23 a 33 anos & 17 & $34 \%$ \\
\hline 34 a 44 anos & 27 & $54 \%$ \\
\hline 45 ou mais & 06 & $12 \%$ \\
\hline Com quem vive & & \\
\hline Cônjuge e filhos & 35 & $70 \%$ \\
\hline Pais, filhos e irmãos & 11 & $22 \%$ \\
\hline Sozinho e outros & 04 & $8 \%$ \\
\hline Total & 50 & $100 \%$ \\
\hline
\end{tabular}

Fonte: pesquisa de campo, Vitória da Conquista - Bahia, 2016.

O público feminino tem sido cada vez mais incidente nos grupos de pesquisa Lopes (2005) "A predominância feminina no cuidado faz com que ainda se identifique um discurso homogêneo em relação ao sexo".

. No estudo realizado constatou-se que houve uma maior predominância de profissionais de saúde do sexo feminino (88\%), casados (62\%), ensino médio (40\%), com idade de 34 a 44 anos e que moram com o cônjuge e com os filhos (70\%) conforme dados da tabela 1 (profissionais de saúde entrevistados).

Na pesquisa de Kirchhog et al. (2008) a faixa a maioria (36,7\%) com idade entre 22 e 38 anos. Esse resultado demonstra que a prevalência dos profissionais dessas idades encontradas, demonstra estes se encontram em uma idade no qual já tiveram a oportunidade de aprendizado ao longo dos anos. Muitos possuem família constituída, com filhos, marido e todas as atribuições que a idade encontrada pode oferecer. 
Na pesquisa de Martins (2002) o percentual de casados foi de 58,47\%. Nesta análise foi observado que a profissão exige muito do profissional e muitos destes preferem não tem tempo de manter uma vida social em atividade. Isso acaba interferindo diretamente nos relacionamentos de muitos, tornando cada vez mais difícil o seu convívio com outros seres humanos.

De acordo as informações coletadas no periódico da Expor (2012) o estudo é tido como algo extremamente importante para vida de todo cidadão, sendo ferramenta indiscutível que fornecerá condições de sobrevivência, tornando-nos mais forte, ágeis e observadores de oportunidades. Entendendo este conceito é possível determinar que nada funciona de forma adequada se o individuo não tiver educação escolar, pelo menos a básica.

TABELA 02: Hábitos de vida diária

\begin{tabular}{l|c|c}
\hline \multicolumn{1}{c|}{ VARIÁ VEL } & N & \% \\
\hline Número de horas semanais de trabalho & 39 & $78 \%$ \\
20 a 40 horas & 06 & $12 \%$ \\
41 a 60 horas & 04 & $4 \%$ \\
Mais de 60 horas & & $14 \%$ \\
Etilismo & 07 & $24 \%$ \\
Indicação de Alcoolismo & 12 & $28 \%$ \\
Não Ingere bebida alcoólica & 14 & $34 \%$ \\
Sugestivo de Alcoolismo & 17 & $26 \%$ \\
Não há problemas com bebida & & $74 \%$ \\
Você estudou em & 13 & $96 \%$ \\
Escola Particular & 27 & $4 \%$ \\
Escola Pública & & \\
Você trabalha & 48 & $22 \%$ \\
Sim & 02 & $26 \%$ \\
Não & & $10 \%$ \\
Atividade Física & 11 & $22 \%$ \\
Ativo & 13 & $20 \%$ \\
Irregularmente Ativo A & 05 & $60 \%$ \\
Irregularmente Ativo B & 11 & $40 \%$ \\
Muito ativo & 10 & $100 \%$ \\
Sedentário & & \\
Prática atividade física & 30 & 20 \\
Sim & 50 & \\
Não & & \\
\hline Total & & \\
\hline
\end{tabular}

Fonte: pesquisa de campo, Vitória da Conquista - Bahia, 2016. 
Id on Line Revista Multidisciplinar e de Psicologia

Id on Line Multidisciplinary and Psycology Journal

Quando abordados sobre os hábitos de vida dos profissionais de saúde entrevistados chegou-se ao resultado de que $78 \%$ trabalham de 20 a 40 horas semanais, 34\% não apresenta problemas com bebida, 74\% estudaram em escola pública, 96\% trabalham, 26\% realiza atividade física irregularmente ativo A e $60 \%$ praticam atividade física.

Freitas (2006) em seu artigo afirma que a carga horária semanal de trabalho dos profissionais de saúde tende há variar a depender da instituição, onde em alguns lugares são de trinta, trinta e seis, quarenta horas e quarenta e quatro horas semanais, sendo ainda mais comum a jornada de trinta e seis horas. Sabe-se que no local em questão a jornada semanal de trabalho é de 36 horas semanais, contudo existem algumas falhas no que diz respeito à troca de plantões efetuado pelos mesmos e entre si, fazendo com que muito fiquem sobrecarregados. É possível apontar o fato de que muitos fazem essa dupla jornada devido à má remuneração salarial que todas as classes de profissionais de saúde veem recebendo, fazendo com que os profissionais se esforcem um pouco mais para alcançar um salário digno.

Se tratando dos profissionais de saúde a ausência de alguns benefícios tem interferido diretamente na sua conceituação voltada para a qualidade de vida, pois um dos maiores prejuízos relatados nos estudos é o ritmo de vida nos quais muitos se encontram inseridos, prejudicando assim toda a sua relação pessoal para com os seus familiares (NEVES, et al. 2010).

TABELA 03: Situações que podem interferir o meio

\begin{tabular}{l|c|c}
\hline \multicolumn{1}{c|}{ VARIÁVEL } & N & \% \\
\hline Meio & & $14 \%$ \\
Boa & 03 & $18 \%$ \\
Necessita melhorar & 17 & $68 \%$ \\
Regular & 29 & $40 \%$ \\
Relações Sociais & 20 & $4 \%$ \\
Boa & 02 & $36 \%$ \\
Necessita Melhorar & 18 & $20 \%$ \\
Regular & 10 & \\
Muito Boa & & $8 \%$ \\
Qualidade de vida & 04 & $12 \%$ \\
Boa & 06 & $80 \%$ \\
Necessita Melhorar & 40 & $10 \%$ \\
Regular & & $12 \%$ \\
Domínio Físico & 05 & \\
Boa & 06 & \\
Necessita melhorar & & \\
\hline
\end{tabular}


Id on Line Revista Multidisciplinar e de Psicologia

Id on Line Multidisciplinary and Psycology Journal

\begin{tabular}{l|l|l}
\hline Regular & 39 & $78 \%$ \\
Domínio Psicológico & & \\
Boa & 07 & $14 \%$ \\
Necessita Melhorar & 09 & $18 \%$ \\
Regular & 34 & $68 \%$ \\
\hline Total & 50 & $100 \%$ \\
\hline
\end{tabular}

Fonte: pesquisa de campo, Vitória da Conquista - Bahia, 2016.

Em se tratando das situações que podem interferir o meio em que, $68 \%$ dos profissionais responderam que o meio pode interferir $40 \%$ relataram que possuem uma boa relação no local onde se encontram inserida, com qualidade de vida regular $80 \%$, domínio físico regular $78 \%$ e domínio psicológico de $68 \%$.

$\mathrm{Na}$ pesquisa de Martins (2002) para 56,15\% dos profissionais consideram que a qualidade de vida interfere em todo o ambiente no qual estão inseridos, realizando as suas atividades normais. Tais informações demonstram que a falta da qualidade de vida tem o poder de influenciar diretamente na vida de todos, pois, muitos necessitam deixar suas residências, filhos, amigos e vida social, para poder exercer a profissão em sua totalidade.

Silva (2011) em seu artigo relata que tem sido difícil para os profissionais principalmente os que atuam, em longos períodos, manter suas relações sociais com sua família e com tudo que possa lhes proporcionar uma vida de qualidade.

Quando o serviço é realizado em equipe, observa-se uma satisfação maior dos profissionais, onde os mesmos apresentam sentimentos favoráveis que refletem diretamente na qualidade e adequação dos serviços prestados. Assim, outros aspectos importantes devem ser considerados, tais como a liberdade para tomar decisões, remuneração reconhecida conforme o trabalho realizado e possibilidade de crescimento profissional. No entanto conforme afirma Nunes et al. (2010) a insatisfação dentro do ambiente de trabalho pode desencadear uma série de fatores negativos, podendo assim interferir em todo o processo do cuidar, além de fornecer um desgaste muito grande para toda à equipe. $\mathrm{O}$ autor afirma ainda que algumas estratégias devam ser realizadas na tentativa de minimizar a insatisfação no ambiente de trabalho (NUNES, et al. 2010). 
Id on Line Revista Multidisciplinar e de Psicologia

Id on Line Multidisciplinary and Psycology Journal

TABELA 04: Avaliação dos riscos

\begin{tabular}{l|c|c}
\hline \multicolumn{1}{c|}{ VARIÁ VEL } & N & \% \\
\hline Nível de estresse & 24 & $48 \%$ \\
$0-04$ & 22 & $44 \%$ \\
$05-09$ & 04 & $8 \%$ \\
$10-12$ & & \\
Resultado Estresse & 16 & $32 \%$ \\
A vida pode estar um pouco estressante para você & 04 & $8 \%$ \\
Parabéns seu corpo este em pleno funcionamento & 04 & $8 \%$ \\
Seu nível de estresse está altíssimo & 26 & $52 \%$ \\
Seu nível de estresse está alto & & \\
Depressão & 01 & $2 \%$ \\
Depressão grave & 28 & $56 \%$ \\
Depressão moderada a grave & 21 & $42 \%$ \\
Sem depressão ou depressão leve & 50 & $100 \%$ \\
\hline Total & & \\
\hline
\end{tabular}

Fonte: pesquisa de campo, Vitória da Conquista - Bahia, 2016.

Quando abordados sobre a avaliação dos riscos existentes na profissão $48 \%$ informaram que o nível de estresse encontra-se de 0 -04, com um alto nível de estresse $52 \%$, possuindo um quadro de depressão moderada a grave $56 \%$.

Junior et al. (2006) afirma que são muitas as manifestações que podem ser percebidas nos profissionais de saúde desde as manifestações como insônia, irritabilidade, sonolência excessiva durante o dia e à noite, durante o trabalho, fadiga contínua, e mau funcionamento do aparelho digestivo. Pode-se ainda observar manifestações mais graves, como o aumento do risco de doenças cardiovasculares, inclusive infarto do miocárdio.

\section{Considerações Finais}

São muitas as transformações que vem sendo desencadeadas no mercado de trabalho, principalmente no que se refere ao setor da área de saúde. As mudanças não estão diretamente relacionadas às evoluções tecnológicas desencadeadas ainda pela era industrial, algumas destas têm sido notada no momento em que se observa o grande número de profissionais que atuam na área por baixos salários e péssimas condições de trabalho. 
Com a análise efetuada foi possível observar que os profissionais do local de escolha conceituam a qualidade de vida como algo que esta diretamente relacionada ao aumento salarial, não focando nas condições de trabalho adequado, nas doenças que poderiam se evitadas com uma carga horária digna, assim como o que poderiam esta ganhando com uma oferta de emprego de qualidade. $\mathrm{O}$ conceito de qualidade de vida, portanto, pode ser definido como algo muito subjetivo, impossível de ser avaliado de forma clara. Contudo com esse estudo foi possível alcançar o objetivo proposto. Vale ressaltar ainda a grande necessidade de orientacão para com os mesmos, pois o valor que a vida tem, supera toda e qualquer remuneração sal $\varepsilon$

\section{Referências}

ALMEIDA, M. A. B. Qualidade de vida: definição, conceitos e interfaces com outras áreas de pesquisa, 2012. Disponível em: http://each.uspnet.usp.br/edicoes-each/qualidade_vida.pdf acessado em 15 de novembro de 2016 as 11:34.

BASTOS, A. Conceito básico sobre qualidade de vida, 2011. Disponível em: < http://www.trabalhosfeitos.com/ensaios/Conceito-B\%C3\%A1sico-Sobre-Qualidade-DeVida/96515.html> acessado em 05 de novembro de 2016 as 17:09.

COREN - GO: Conselho Regional de Enfermagem - Goiás. BEZERRA, A. L. Q. A Enfermagem: principais dificuldades na prática e o caminho a ser seguido, 2014. Disponível em: < http://www.corengo.org.br/a-enfermagem-principais-dificuldades-napratica-e-o-caminho-a-ser-seguido_1844.html> acessado em 06 de setembro de 2015 as 19:44.

ELIAS, M. A relação entre o trabalho, a saúde e as condições de vida: negatividade e positividade no trabalho das profissionais de enfermagem de um hospital escola.

Latino-am Enfermagem. 2006; Disponível em:

http://www.scielo.br/scielo.php?script=sci_arttext\&pid=S1414-81452011000200008> acessado em 14 de outubro de 2016 as 09:40.

ELVIRA, M. Definição de qualidade de vida é subjetiva, e daí?, 2013. Disponível em:< http://exame.abril.com.br/revista-voce-rh/edicoes/28/noticias/a-subjetividade-do-estar-bem> acessado em 19 de outubro de 2016 as 10:35.

EXPOR - Mais evolução. A importância dos estudos em nossa vida, 2012. Disponível em: https://exporr.wordpress.com/2012/09/06/a-importancia-dos-estudos-na-nossa-vida/ acessado em 07 de novembro de 2016 as 07:14.

FREITAS, J. S. et al. Qualidade dos cuidados de enfermagem e satisfação do paciente atendido em um hospital de ensino, 2014. Revista Latino Americana de Enfermagem, 
Id on Line Revista Multidisciplinar e de Psicologia

Id on Line Multidisciplinary and Psycology Journal

2014. Disponível em: http://www.scielo.br/pdf/rlae/v22n3/pt_0104-1169-rlae-22-0300454.pdf acessado em 15 de novembro de 2016 as 11:08.

GIL, A. C. Métodos e Técnicas de Pesquisa Social. 4. Ed. São Paulo: Atlas, 2008. Como elaborar projetos de Pesquisa. 5. ed. São Paulo: Atlas, 2010. 184 p.

JUNIOR, A. C. S. et al. O trabalho noturno e a qualidade de vida dos profissionais de enfermagem, 2006. Revista Mineira de Enfermagem, vol. 10-1. Disponível em: < http://www.reme.org.br/artigo/detalhes/383> acessado em 06 de setembro de 2016 as 14:04.

KIRCHHOF, A. L. C. et al. Condições de trabalho e características sóciodemográficas relacionadas à presença de distúrbios psíquicos menores em trabalhadores de enfermagem, 2008. Disponível em: http://www.scielo.br/pdf/tce/v18n2/03.pdf> acessado em 10 de novembro de 2016 as 15:29.

LOPES, M. J. M. A feminização persistente na qualificação profissional da enfermagem brasileira, 2005. Disponível em: < http://iseib.edu.br/biblioteca/wpcontent/uploads/2013/05/AFeminizacaoPesiste.pdf> acessado em 04 de novembro de 2016 as $11: 25$.

MARTINS, M. M. Qualidade de vida e capacidade para o trabalho dos profissionais em enfermagem no trabalho em turnos, 2002. Disponível em: < http://nucidh.ufsc.br/files/2011/09/dissertacao_marilu.pdf> acessado em 09 de novembro de 2016 as $20: 38$.

NEVES, M. J. A. O. et. al. Influência do trabalho noturno na qualidade de vida do enfermeiro, 2010. Revista de Enfermagem UERJ - Universidade Estadual do Rio de Janeiro, $2010 \quad-\quad$ jan/mar; 18(1):42-47. Disponível em: < http://www.facenf.uerj.br/v18n1/v18n1a08.pdf> acessado em 06 de setembro de 2016 as 18:29.

NUNES, C. M. et. al. Satisfação e insatisfação no trabalho na percepção de enfermeiros de um hospital universitário, 2010. Revista Eletrônica de Enfermagem. Disponível em: https://www.fen.ufg.br/fen_revista/v12/n2/v12n2a04.htm acessado em 18 de novembro de 2016 as $17: 31$

OMS - Organização Mundial da Saúde. Conceito de Saúde segundo a OMS/WHO. CMEI Câmara dos Especialistas em Medicina Interativa, 2016. Disponível em: < http://cemi.com.pt/2016/03/04/conceito-de-saude-segundo-oms-who/> acessado em 19 de novembro de 2016.

PEREIRA, E. F. Qualidade de vida: abordagens, conceitos e avaliação, 2012. Rev. bras. Educ. Fís. Esporte, São Paulo, v.26, n.2, p.241-50, abr./jun. 2012. Disponível em: http://www.scielo.br/pdf/rbefe/v26n2/07.pdf acessado em 03 de abril de 2015 as 18:18.

POLIT, D. F. BECK, C. T. HUNGLER, B. P. Fundamentos de Pesquisa em Enfermagem,

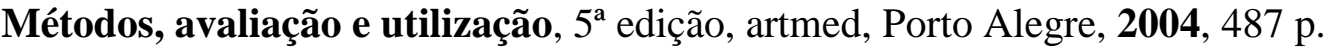


SILVA, R. M. et al. Trabalho noturno e a repercussão na saúde dos enfermeiros, 2011. Esc. Anna Nery vol.15 no. 2 Rio de Janeiro Apr./June 2011. Disponível em: http://www.scielo.br/scielo.php?script=sci_arttext\&pid=S1414-81452011000200008> acessado em 14 de abril de 2015 as 10:06.

\section{Como citar este artigo (Formato ABNT):}

GOMES, R.S.; COQUEIRO, J.F.R. Qualidade de Vida Relacionada à Carga de Trabalho dos Profissionais de Saúde com enfoque nos problemas desencadeados . Id on Line Revista Multidisciplinar e de Psicologia, Janeiro de 2017, vol.10, n.33, p. 249-261. ISSN: 1981-1179.

Recebido: $02 / 12 / 2016$

Aceito: 02/12/2016 